\title{
Parking entrance control using license plate detection and recognition
}

\author{
Mohamed S. Farag, M. M. Mohie El Din, H. A. El Shenbary
}

Department of Mathematics, Faculty of Science, Al-Azhar University, Egypt

\begin{tabular}{l}
\hline Article Info \\
\hline Article history: \\
Received Dec 15, 2018 \\
Revised Jan 21, 2019 \\
Accepted Mar 1, 2019 \\
\hline
\end{tabular}

\section{Keywords:}

Intelligent transportation systems

License plate detection

Recognition

Segmentation

Smart parking

\begin{abstract}
There is no doubt that car parking is a very challenging and interesting topic of surveillance. In the recent years, a lot of smart systems for parking lot access control were developed to control and register the car data. The aim of this paper is to use image processing methods to control the entrance of a smart parking. The steps of car plate recognition are: preprocessing, License plate detection, character extraction and recognition. In the step of preprocessing, image was enhanced and noise was reduced. After preprocessing stage, color filter was used to detect the plate region. In case of large image size DWT was used for feature extraction and decreased the time of the detection stage. In the stage of character segmentation, the image is converted from grayscale to binary according to a given threshold. Filtering the binary image after using the morphological operation method, the largest objects are determined as the segmented plate characters. Finally, the correlation method was used to recognize the segmented characters. In case of similarity, SVM was used as a good classifier. Experimental results using matlab software, view that the proposed method increase the plate detection and recognition rates. It achieved average 97.8\% detection rate, $98 \%$ segmentation rate and $97 \%$ recognition rate, so it will be a good method for smart parking entrance control.
\end{abstract}

Copyright $\odot 2019$ Institute of Advanced Engineering and Science. All rights reserved.

\section{Corresponding Author:}

Mohamed S. Farag,

Department of Mathematics,

Faculty of Science, Al-Azhar University,

Nasr city, 11884, Cairo, Egypt, Tel: 0020-1006-574-243.

E-mail: mohamed.s.farag@azhar.edu.eg

\section{INTRODUCTION}

The industrialization of the world, slow paced city development, and increase in number of cars has resulted in parking problems. There is a need for a robust system to be used for searching the free parking lots. Smart Parking System (SPS), is considered to be a small version of an Intelligent Transportation Systems (ITS) [1]. This SPS using Internet of Things (IoT), to reduce the parking congestion.

In [2] authors introduced a method for parking guiding system and space analyzing. IOT begin with things which have independent connected devices. These devices should be monitored, or under controlled using connected computers [3]-[4]. We have created a smart parking guidance algorithm in [5] depending on a cost function. In order to identify the cars, image processing techniques also was introduced. To detect the illegal (unreserved) cars, they used Optical character recognition (OCR) method. OCR is used to convert the row text and documents (like text books) into electronic readable les like (pdf). In [6] a hybrid feature extraction method was introduced for facial recognition and gave average recognition rate $100 \%$.

An automatic car parking system using FPGA based on emergency conditions was proposed to detect the drivers condition and perform specific tasks such as warn the drivers [7]. We want to use image processing techniques in order to control the parking entrance. In order to solve the problem of parking 
entrance management, a lot of research was made. Automatic License Plate Detection(ALPD) is to automatically detect the license region from a given image.

In [8] an ALPD method consist of five steps was introduced for handling different hazardous rates. The previous ALPD was developed and tested on database consists of 850 images in different controlled conditions like night, indoor, blurry, day, rainy, foggy and tilted LP. This method achieve global $94 \%$ detection rate, but it can be enhanced by using a lot of filtering methods.

In [9] a new method presented to apply the LPR systems for Iranian license plates. Increasing the accuracy of the character recognition phase rate and decreasing the training rate are the main advantages of the new hybrid model. The K-NN was implemented as the first classification method; the confusion problem related to similar characters in the license plates was overcome by using the multiple SVMs classification model. The SVMs has improved the performance of the K-NN in the recognition of similar characters. The SVMs was trained and tested only for the similar characters. The proposed method gave $97 \%$ character recognition rate.

\section{RESEARCH METHODS}

\subsection{Morphological Operation}

Morphological of image preprocessing is a sequence of non-linear operations depending on the morphology or shape of the features. Morphological operation does not depend on the numerical values, but depends on the related ordering of the pixel values and so are especially effective to the preprocessing of binary images. We want to thin connectivities between large white areas extended outer region of given images and remove small thin white objects. Due to the previous needs, morphological operation [10] (erosion, then dilation) is applied on a given binary image $f$. In the erosion step: erosion image $f$ by structuring element $s$ output a new image $g$ having values one in all pixels $(x ; y)$ of a structuring element's when $s$ fits $f$. In the dilation step: dilation of a binary image $f$ by a structuring element $s$ output a new image $g$ having values one in all pixels $(x ; y)$ of a structuring element's when s hits f. Figure 1 show an example of hitting and fitting a binary image.

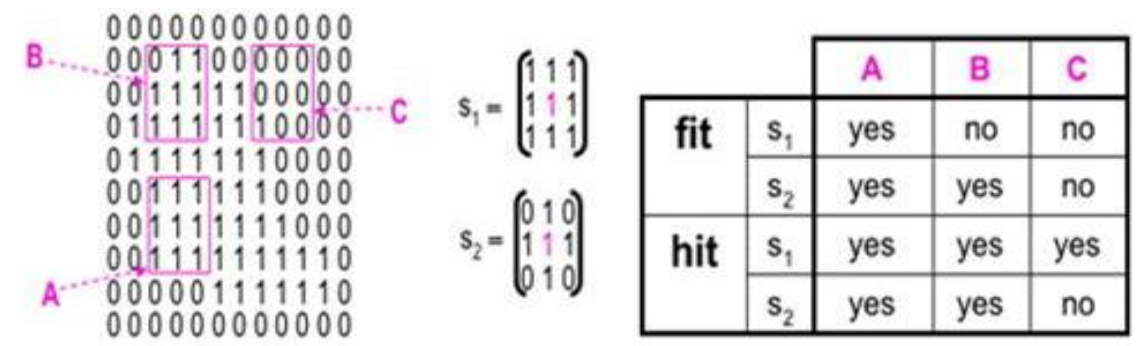

Figure 1. Example of hitting and fitting a binary image

\subsection{Discrete Wavelet Transform}

Wavelet transforms are mathematical functions used to convert data into a lot of frequency components and each component is studied with a resolution corresponding to its scale. Wavelets were presented independently in the field of mathematics [11], electrical engineering and quantum physics. In the last decades, many new wavelet applications were appeared like image compression, prediction of earthquakes, turbulence, radar and human vision. The mother wavelet decomposition function for an image is defined as:

$$
\Psi_{u, v(t)}=\sum_{x=0}^{N-1} \sum_{y=0}^{N-1} f(x, y) \exp ^{-j \pi \frac{(u x+v y)}{N}}
$$

Where,

$\exp ^{-j \pi \frac{(u x+v y)}{N}}$ is a Kernel function, $f(x, y)$ is a2Dimage, and $N$ is count of pixels.

The Wavelet transform is a useful computational tool for applications of signal and image processing [12]. DWT is used in a wide range in pattern recognition area [13], [14]. 
Figure 2 view an example of DWT one level decomposition. LL1 describes approximate information, LH1 describes Horizontal information, HL1 describes vertical edge information, and finally the diagonal information is presented by $\mathrm{HH} 1$.

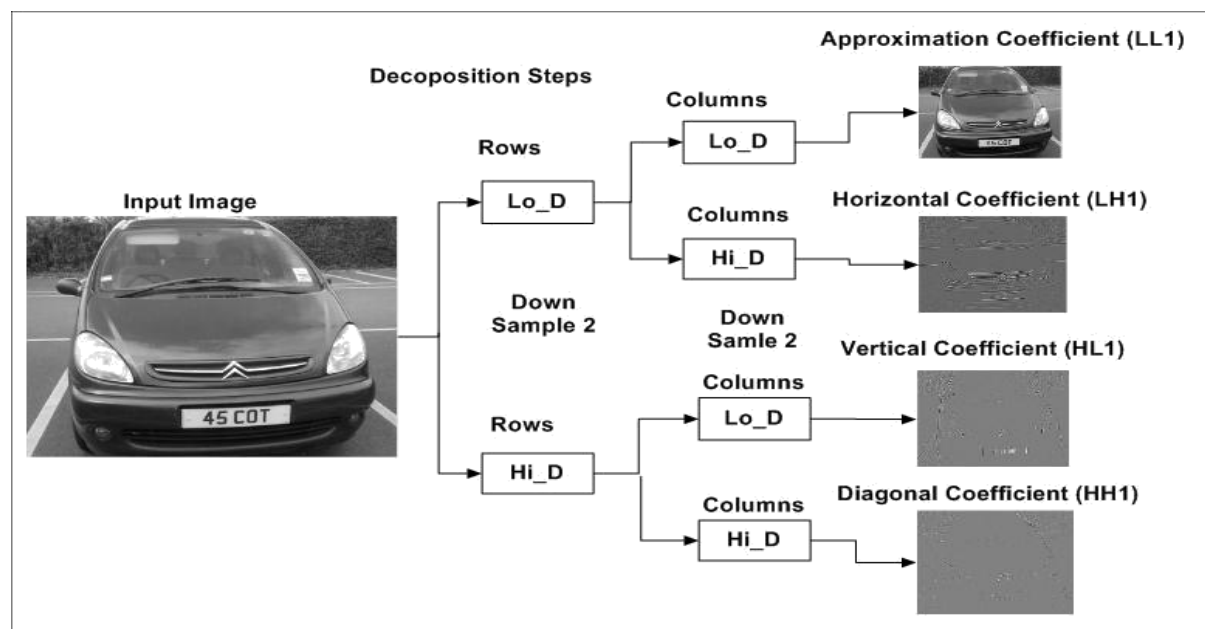

Figure 2. DWT coefficients

\section{PROPOSED METHOD}

This paper presents a hybrid framework for robust license plate detection and recognition system. Figure 3 view the general steps of ALPR system.

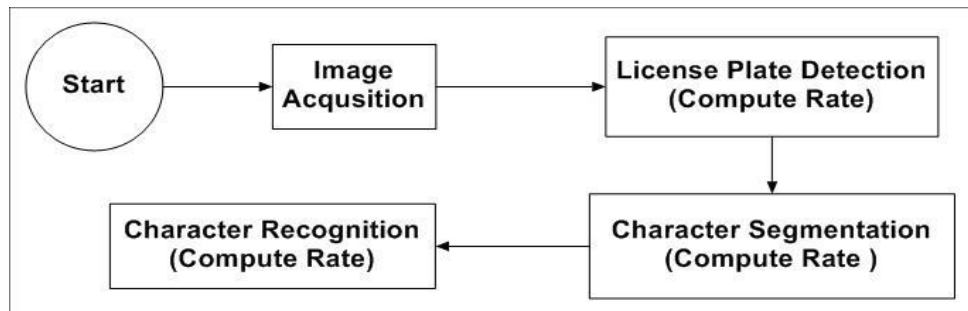

Figure 3. General steps of ALPR system

\subsection{Image Acquisition}

Figure 4 show how to get the car image. A digital camera is installed in the entrance of the parking, to collect the car image. Once the image is captured, we directly go to the next step (Plate Detection).

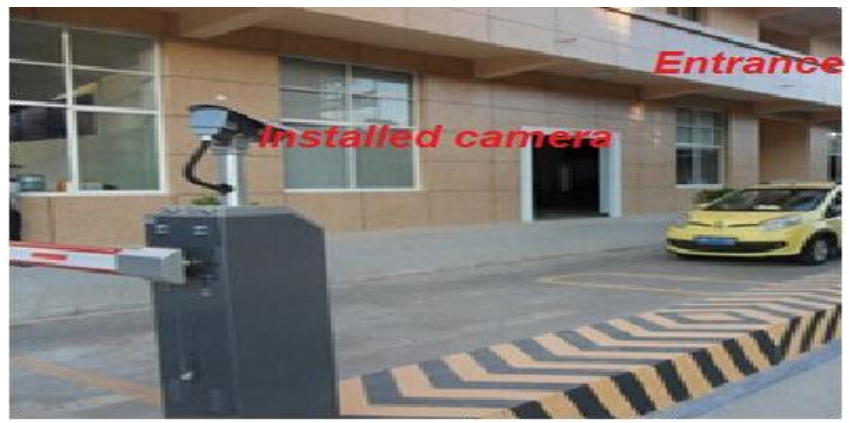

Figure 4. Image acquisition 


\subsection{Plate Detection}

Plate area extraction or detection is the most important step because the license plate is in a small region and may be anywhere in the given image. It can be defined by its features. The proposed detection method depends on the License Plate color. The goal of the detection step is to mark an area with highest probability of having the plate. The detection method depends on two License Colors(White and Yellow backgrounds). The given RGB Image is converted to index image using rgb2ind() function, then use two color filter. White range is $(\mathrm{R}>0: 5 ; \mathrm{G}>0: 3 ; \mathrm{B}>0: 81)$ and Yellow range is $(\mathrm{R}>0: 3 ; \mathrm{G}>0: 3 ; \mathrm{B}<0: 31)$. Now we shall remove the noise objects using bwareaopen() function to remove objects smaller than 200. Using the morphological operation for both erosion and dilation. Remove objects smaller than 3500 pixel, then the area with the biggest size is determined as the plate area. Figure 5 view example of detected plates with two colors yellow and white backgrounds.

The detected area is converted from rgb to gray to be used in the stage of character segmentation due to the following (2).

$$
Y=0.2989 * R+0.5870 * G+0.1140 * B
$$

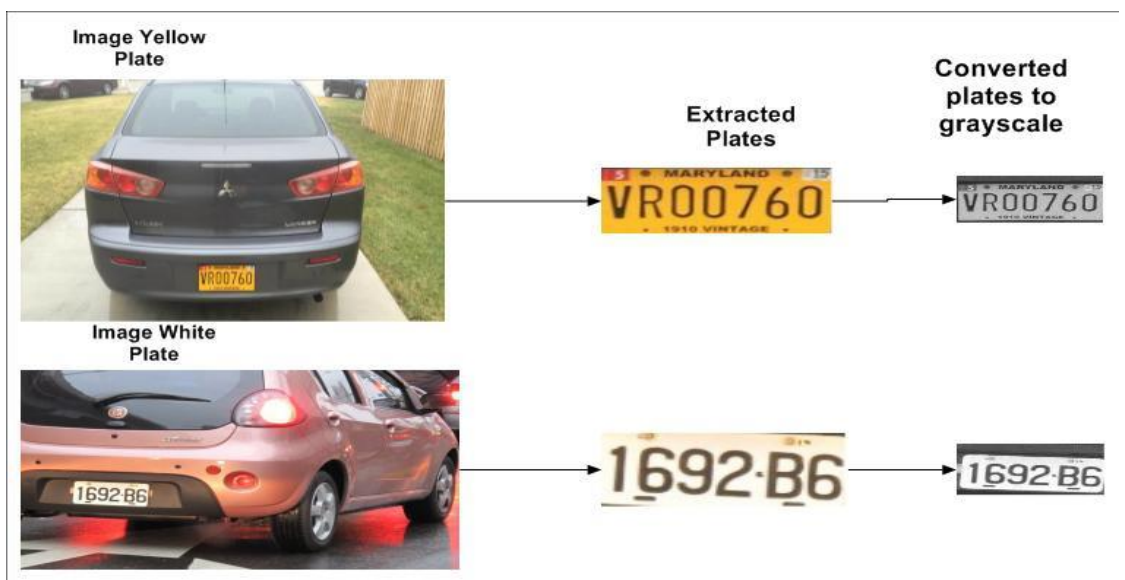

Figure 5. Detected plates

In case of white or yellow foreground car, the areas with the smallest size are extracted. the detected areas are not considered to be the correct plate unless the segmentation stage is applied.

In this case, the segmentation stage is used to classify the detected areas. The area that contains characters is considered as the correct plate area. Figure 6 view example of detected plates with two colors yellow and white.

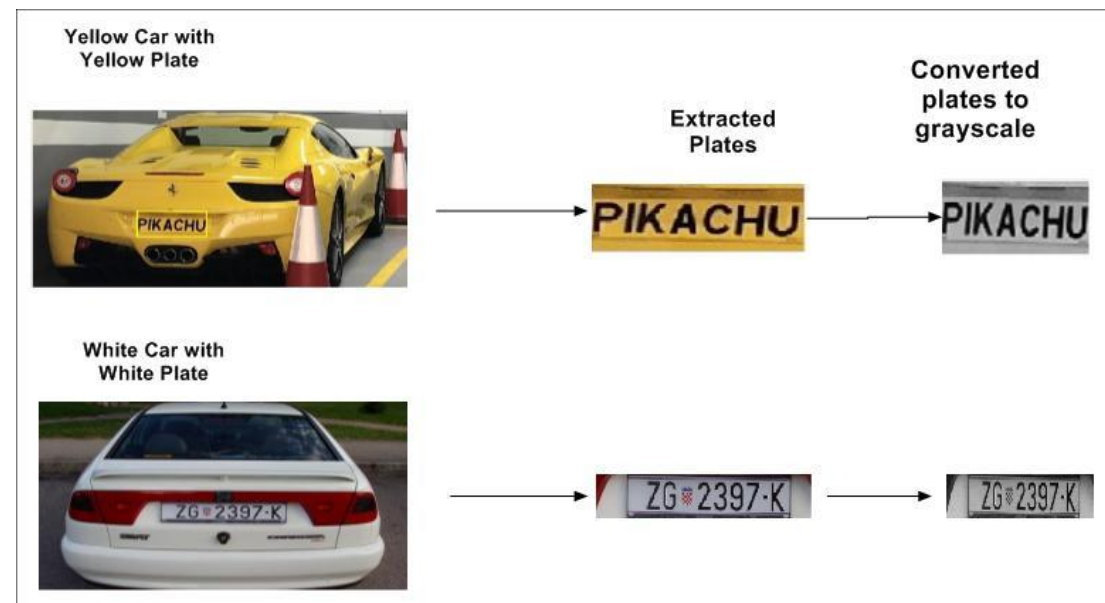

Figure 6. Detected plates in yellow and white foreground 


\subsection{Character Segmentation}

In this stage we have the extracted gray plate and want to segment the characters. Figure 8 view the Steps of the Segmentation stage. The given plate image is converted from grayscale to BW (black and white), based on a threshold corresponding to the following.

$$
Y_{i j}=\left\{\begin{array}{l}
1 \text { if } x_{i j}>\theta \\
0 \text { Othewise }
\end{array}\right.
$$

Using two filters steps:

a. Remove the smallest objects in the binary image, and leave the largest 6 objects.

b. Remove objects which are not in the same vertical range.

Figure 7 view object to be removed, because it is not in the same vertical range. Finally, the segmented character is assigned to the final step (Character Recognition).

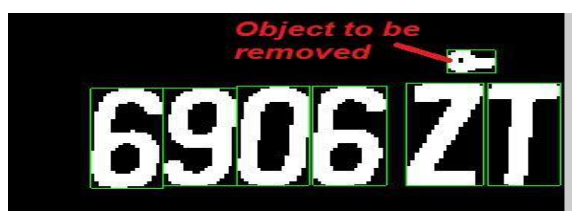

Figure 7. Removed objects

The following algorithm is a pseudo code defines how to remove objects, which are not characters.

Algorithm 1: Pseudo Code for Removing non Characters

\footnotetext{
Require: Objects, Obj_num, Obj_rowrange

Ensure: New Objects

1: for $\mathrm{i}=1$ : Obj_num do

2:Gét Objects i height andwidth

3:if Objects i_width > Objects i_heightthen

4:if Objects i_rowrange! $=\mathrm{Obj}_{-}$_rowrangethen

5:Remove Objects_i from NewŌbjects

6:endif

7:endif

8: endfor
}

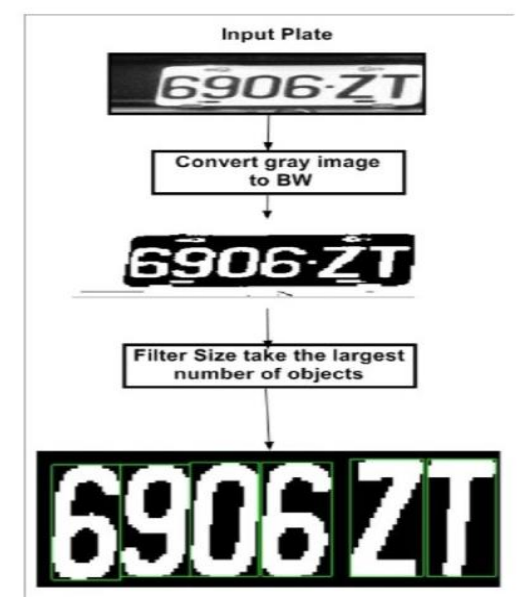

Figure 8. Segmentation process 


\subsection{Character Recognition}

In this step, we have the segmented characters and want to recognize it. The following (3) was used for computing correlation rate between two images A and B.

$$
r=\frac{\sum_{i} \sum_{j}\left(A_{i j}-\bar{A}\right)\left(B_{i j}-\bar{B}\right)}{\sqrt{\left(\sum_{i} \sum_{j}\left(A_{i j}-\bar{A}\right)^{2} * \sum_{i} \sum_{j}\left(B_{i j}-\bar{B}\right)^{2}\right)}}
$$

Where both A and B are the mean of A and B respectively. In case of similarity, which cannot be recognized using the correlation method. For example (D, O and 0$)$, (B and 8$)$ and (2 and $\mathrm{z}$ ), in this case further training is needed. The way is to use Support vector machine (SVM), which is defined as a learning method used for data classification. SVM considered as a statistical classification algorithm and was proposed by Cortes and Vapnik [15]. SVM becomes popular because it gave high rate in handwritten digit classification. SVM was used for image classification in [16]. This algorithm search for the maximum separating hyperplane, which is defined by the hyperplane with the maximum distance between the training tuples.

\section{RESULTS AND DISCUSSION}

In this experiment, we test the accuracy and efficiency of the system. The white color plates images are taken from AOLP database [17] cited in paper [18]. The yellow color plates are collected from the internet. The car images database has different conditions, like brightness, different font size, shadow, low contrast, different car colors, different font type and different images size. Table 1 view the experimental results of the detection step. In case of white background the correct detection rate was $97.4 \%$, and in case of Yellow plate the correct rate was $97.8 \%$.

Table 1. Experimental Plate Detection Result

\begin{tabular}{ccccc}
\hline LP Type & Number of Images & Success Number & Success Rate & Average Image Size(Pixel) \\
\hline White-Black & 117 & 114 & 97.4 & $640 * 480$ \\
Yellow-Black & 46 & 45 & 97.8 & $1024 * 768$ \\
Total & 163 & 159 & 97.5 & $\ldots$. \\
\hline
\end{tabular}

Table 2 show the comparison between the proposed detection method and other reported papers. The reported papers used the same database, and we add the database of yellow color plates. It is clear that the proposed method outperform the reported method in both correct detection rate and execution time per each image.

Table 2. Comparison with the Reported Articles

\begin{tabular}{|c|c|c|c|c|}
\hline No & Reference & $\begin{array}{l}\text { Correct } \\
\text { Rate }\end{array}$ & $\begin{array}{l}\text { Average Execution } \\
\text { Time(Sec.) }\end{array}$ & System Specification \\
\hline 1 & Azam et al [19] & 86.15 & 0.45 & $\begin{array}{c}\text { C: Intel Core } 2 \mathrm{Duo}, 2.2 \mathrm{GHz} \\
\text { S: } 120 \mathrm{X} 40 \text { (non-tilted), } 80 \text { X55 (tilted) }\end{array}$ \\
\hline 2 & Ghahnavieh et al [20] & 95.5 & 0.0968 & $\begin{array}{l}\text { C: Core } 2 \text { Quad, } 2.67 \mathrm{GHz} \text { S: } \\
640 \times 480 \text { to } 2000 \times 1500\end{array}$ \\
\hline 3 & Tadic et al [21] & 94.4 & - & - \\
\hline 4 & Panahi et al [22] & 96.3 & 0.18 & $\begin{array}{c}\text { C: Intel Core i5, } 2.2 \mathrm{GHz} \text { S: } 16 \mathrm{X} 80 \\
\text { to } 25 \mathrm{X} 1508\end{array}$ \\
\hline 5 & Proposed Method & 97.5 & 0.123 & $\begin{array}{l}\text { C: Intel Core i3, } 2.4 \mathrm{GHz} \text { S: } \\
640 * 480 \text { to } 1024 * 768\end{array}$ \\
\hline
\end{tabular}

In case of large image size (for example: $2000 * 1950$ pixel), DWT was used before detection stage. DWT generate 4 coefficients (approximation, horizontal, vertical, diagonal). The approximation coefficient was used as the test image, then the detection step was applied. Using DWT decrease the time of the detection stage from 0.4892 seconds to 0.167 , approximately $67 \%$ decrease time rate.

Applying character segmentation stage on the detected plates, the experimental result gave $98 \%$ correct segmentation rate.

For recognition, pattern with maximum correlation is used as the recognized pattern. In case of similarity, the MultiSvmTrain() function was used to models a given training set with a corresponding group vector and classifies a given test set using MultiSvmClassify() function according to a one versus all relation. 
Each character in training set has 3 images. The different Kernel functions test results showed that the best SVMs function for this study is the Gaussian Radial Basis Function (RBF). The RBF kernel is defined as:

$$
k_{R B F}\left(x, x^{\prime}\right)=\exp \left[-\gamma\left\|x-x^{\prime}\right\|^{2}\right]
$$

where is a scaling parameter that sets the spread of the kernel and its default value is 1 . The characters which were considered as similar characters $(0, \mathrm{D}, \mathrm{O}),(\mathrm{B}, 6,8),(\mathrm{S}, 5)$ and $(2, \mathrm{Z})$, SVM was used to buildthe model for each group. If the correlation method gave any characters of them, the MultiSvmClassify() will used to classify the given characters using its group model. SVM gave average $97 \%$ recognition rate, unlike the correlation method gave $94 \%$ correct recognition rate.

\section{CONCLUSION}

In this paper we present a hybrid method to improve on the current techniques of car plate detection and recognition. The proposed method detects the plate number in two background color (White and Yellow). The detection rate was increased to $97.8 \%$. In case of big image size the DWT was used in the preprocessing stage, which decreased the time consumed in detection stage with $67 \%$. In the segmentation stage, plate converted from grayscale to black and white. A defined filter was used to remove objects which are not characters. Objects are not in the same row range, or the width is larger than height. Finally the correlation method was used to recognize the segmented characters. If the character is defined to be $(\mathrm{D}, 0, \mathrm{O}, \mathrm{B}, 8, \mathrm{Z}, 2$...) which has similarity, in this case SVM used for classification. SVM gave $97 \%$ recognition rate and correlation method gave $94 \%$ recognition rate, which means that using SVM is essentially for increasing recognition rate.

\section{REFERENCES}

[1] F. S. A. Mahmud, G. M. Khan, M. Rahman, and H. Zafar. "A Survey of Intelligent Car Parking System". Journal of Applied Research and Technology, 2013, vol.11, pp. 714 - 726.

[2] C. Shi, J. Liu, and C. Miao. "Study on Parking Spaces Analyzing and Guiding System Based on Video". IEEE, 23rd International Conference on Automation and Computing (ICAC), 2017, pp. 1 - 5.

[3] A. Khanna and R. Anand. "IoT Based Smart Parking System". International Conference on Internet of Things and Applications (IoTA), Maharashtra Institute of Technology, Pune, India, 2016, pp. 266 - 270.

[4] T. H. Ham, M. Tsai, D. B. Nguyen, C. Dow, and D. Deng. "A Cloud-Based Smart-Parking System Based on Internet-of-Things Technologies". The journal for rapid open access publishing, 2015, vol. 3, pp. 1581 - 1591.

[5] M. S. Farag, M. M. Mohie El Din, and H. A. El Shenbary. "Smart Parking Guidance Using Optimal Cost Function”. Computer and Information Science, Published by Canadian Center of Science and Education, 2017, vol.10, No. 1, pp. 48 - 53.

[6] H. Ali, M. Hariharan, S.Yaacob, and Abdul Hamid Adom. "Hybrid Feature Extraction for Facial Emotion Recognition”. Int. J. Intelligent Systems Technologies and Applications, 2014, Vol. 13, No. 3, pp. 202 - 221.

[7] K. Jing Yong and Muataz H. Salih. "Design and Implementation of Embedded Auto Car Parking System Using FPGA for Emergency Conditions". Indonesian Journal of Electrical Engineering and Computer Science, 2019, Vol. 13, No. 3, pp. 876-883.

[8] S.Azam and MdMonirul Islam. "Automatic License Plate Detection In Hazardous Condition”. Journal of Visual Communication and Image Representation, 2016, vol. 36, No. Supplement C, pp. 172 - 186.

[9] SS. Tabrizia and NadireCavus. "A Hybrid Knn-Svm Model for Iranian License Plate Recognition". 12th International Conference on Application of Fuzzy Systems and Soft Computing, (ICAFS), published by ELSEVIER, 2016, pp. 588594.

[10] M. Goyal. "Morphological Image Processing". International Journal of Computer Science and Technology, 2011, vol. 2, No. 4, pp. $161-165$.

[11] R. S. Sabeenian. "Hand Written Text to Digital Text Conversion Using Radon Transform and back Propagation Network (RTBPN)". International Journal of Computers Information Technology and Engineering (IJCITAE), 2010, Vol. 101, pp. 498 - 500.

[12] Ch. SrinivasaRaoh V. Ashok Kumar, C. Dharmaraj. "A Hybrid Digital Watermarking Approach Using Wavelets and LSB". International Journal of Electrical and Computer Engineering (IJECE), 2017, Vol. 7, No. 5, pp. 2483-2495.

[13] M. M. MohieEl-din, N. I. Ghali, A. G. Ahmed, and H. A. El-Shenbary. "A Study on the Impact of Wavelet Decomposition on Face Recognition Methods". International Journal of Computer Applications (IJCA), 2014, vol. 87, no. 3, pp. $14-21$.

[14] M. M. MohieEl-din, M. Y. El-Nahas, and H. A. El-Shenbary. "Hybrid Framework for Robust Multimodal Face Recognition". International Journal of Computer Science Issues (IJCSI), 2013, vol. 10, Issue 2, no. 2, pp. $471-476$.

[15] C. Cortes and V. Vapnik. "Support Vector Networks. Machine Learning”, 1995, vol. 20, no. 3, pp. 273 - 297. 
[16] Y. Khalid Zamil, S. A. Ali, and M. Abdullah Naser. "A Spam Image Email Filtering Using K-Nn and SVM". International Journal of Electrical and Computer Engineering (IJECE), 2019, Vol. 9, No. 1, pp. 245-254.

[17] LPR Database [online]. Available from: "http://aolpr.ntust.edu.tw/lab/download.html".

[18] G. S. Hsu, J. C. Chen, and Y. Z. Chung. “Application-Oriented License Plate Recognition”. IEEE Transactions on Vehicular Technology, 2013, vol.62, no.2, pp. 552 - 561.

[19] S. Azam and M. M. Islam. "Automatic License Plate Detection in Hazardous Condition". Journal of Visual Communication and Image Representation, 2016, vol. 36, pp. 172186.

[20] A. E. Ghahnavieh, A. Amirkhani-Shahraki, and A. A. Raie. "Enhancing the License Plate Character Recognition Mehods by Means of SVM". 22nd Iranian Conference on Electrical Engineering, 2014, pp. 220225.

[21] V. Tadic, M. Propovic, and P. Odry. Fuzzifiedgabor Filter for License Plate Detection". Engineering Applications of Artificial Intelligence, 2016, vol. 48, pp. 40 - 58.

[22] R. Panahi and I. Gholampour. "Accurate Detection and Recognition of Dirty Vehicle Plate Numbers for HighSpeed Applications”. IEEE Trans. Intell. Transp. Syst., 2016, pp. 1 - 13. 